\title{
El cuento de las matemáticas
}

\author{
Diana Margarita Hernández Pacheco* \\ Martha Leidy Hernández Moreno"** \\ José Francisco Leguizamón Romero ${ }^{* * * * *}$
}

Artículo de Investigación

Fecha de Recepción: 29 noviembre 2017.

Fecha de Aprobación: 30 mayo 2018.

\section{Resumen}

En el documento se presenta los resultados de la investigación que tenía como objetivo desarrollar la comprensión lectora en el aula de matemáticas, a través del cuento, en el grado décimo A de la Institución Educativa Magdalena de Sogamoso. Se emplearon los niveles de comprensión lectora expuestos por Barret (1968): Literal, inferencial, crítico y apreciativo, adaptados hacia las matemáticas, utilizando el cuento como mediación, recurso y estrategia. Este proyecto investigativo tuvo un enfoque cualitativo, con la metodología de la investigación acción. Los instrumentos empleados para la recolección de datos fueron pruebas documentales (diagnóstica, intermedia y final), y diarios de campo. Los resultados evidenciaron que, al utilizar el cuento como recurso, el estudiante logró comprender lo que se le preguntaba, relacionar los temas que ya conocía con los nuevos, que el texto le presentaba, realizar razonamientos matemáticos y, por último, usó la temática aprendida en los diferentes contextos para dar solución a situaciones problema planteadas.

Palabras clave: Comprensión, lectura, cuento, aula de Matemáticas, resolución de problemas.
* Institución Educativa Magdalena, Boyacá-Colombia ing_dianamher@hotmail. com

** Institución

Educativa Magdalena, Boyacá-Colombia

marthikhernandez2014@ hotmail.com

**** Universidad Pedagógica y Tecnológica de Colombia, Boyacá-Colombia

francisco.leguizamón@uptc. edu.co

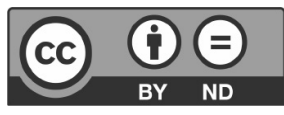




\section{Introducción}

Las pruebas SABER realizadas en Colombia, están enfocadas a evaluar el desempeño de los estudiantes en las diferentes asignaturas, teniendo en cuenta lo que deben alcanzar los estudiantes en un determinado grado, especialmente en las áreas de Castellano y Matemáticas. A partir de un estudio previo, se determinó que era necesario proponer estrategias que contribuyan al fortalecimiento de la compresión lectora y de relacionarla con otras asignaturas, específicamente, con las Matemáticas. En este artículo, se presentan los resultados de una investigación donde se propone el uso del cuento como recurso para fortalecer y desarrollar los niveles de comprensión lectora en matemáticas. Cuando se habla de niveles de comprensión lectora, se constata que ha sido un tema de estudio desde hace mucho tiempo, la mayoría de autores se han basado en la taxonomía propuesta por Barret (1968).

La comprensión lectora es una de las bases más importantes de la educación y debe ser abordada en todas las asignaturas, trabajada de modo transversal, independiente del grado en el que se encuentre el estudiante. Es evidente que, cuando se habla de ella, se le asocia solo con el área de Lengua Castellana, pues ha sido en esta en la que se ha trabajado de manera explícita hasta el momento. Aun así, se sabe que la lectura comprensiva se hace necesaria en todas las áreas, ya que, para aprender, lo primero es comprender. Un lector comprende un texto cuando puede encontrarle significado, cuando puede ponerlo en relación con lo que ya sabe y con lo que le interesa. Pinzás (1995) define la comprensión lectora como el proceso por el cual se emplean las claves dadas por el autor y el conocimiento previo que el lector tiene, para conocer el significado que aquel intenta transmitir. Es decir, si el lector logra conectarse con el autor mediante lo que ya sabe, va a construir un modelo de una manera rápida y detallada, comprendiendo de esta manera lo que está leyendo.

Indicadores como el Programa para la Evaluación Internacional de Alumnos (PISA), demuestran que en Colombia en el año 2016, los estudiantes de las instituciones educativas colombianas, presentaban dificultades en el aprendizaje, especialmente de las áreas de matemáticas y lenguaje. Así mismo, se identificó que cuando se habla de matemáticas en el aula, se presenta un rechazo generalizado, esto se debe a que los docentes aún manejan una enseñanza tradicional donde son los únicos dueños del conocimiento (Porlán, 1993). Además, existe una idea que el docente de castellano es el encargado de enseñarles a los estudiantes lo referente con la comprensión lectora; y el profesor de matemáticas, lo concerniente con el lenguaje matemático, sin tener en cuenta que debe existir una relación entre estos dos tipos, para que los estudiantes alcancen niveles aplicables en su diario vivir (Alonso, Parada, \& Chaparro, 2016).

En general, los estudiantes, en lugar de estar atentos a descubrir nuevos conceptos, a emplear la lógica de cómo 
aplican lo que aprenden en su vida, en el proceso de los razonamientos y la comprensión de los contenidos vistos en clase, se limitan solo a tomar apuntes, a entender la parte mecánica del conocimiento y a automatizarse en el saber tratando de memorizar los conceptos a estudiar. Esto, de cierta manera, se debe a la forma como estos se presentan a los estudiantes, no permitiéndoles construir sus propias ideas y, en especial, el ser capaces por sus propios medios de ponerlos en acción en situaciones fuera del contexto matemático.

Algunos estudios reflejaban la importancia de emplear recursos innovadores para fortalecer la comprensión en matemáticas; entre estos, encontramos a Salas (2012) quien se planteó dos objetivos: conocer y describir los logros y dificultades de los alumnos del nivel medio superior, en relación con la comprensión lectora. En el estudio se proponen cinco etapas de desarrollo (cuestionario, taller con cuestionario, taller literario, resumen y evaluación) por medio de las cuales realizó un diagnóstico del nivel de comprensión lectora de los estudiantes y, así, analizó diferentes estrategias para que los alumnos identificaran las diferentes actividades que ponen en práctica al momento de la comprensión de textos.

Para abordar el uso del cuento como recurso motivador en las matemáticas, se encontró a Marín (1999), en su estudio planteó como objetivo demostrar que la utilización del cuento como herramienta didáctica de aprendizaje, era un acierto en la enseñanza de las matemáticas, despertando así el interés y la motivación de los mismos. En el estudio exponía, de manera detallada, por qué se debían emplear los cuentos para aprender las matemáticas: qué clase de cuentos se debían emplear proponiendo tres clases (cuentos repetitivos, cuento ex profeso y cuentos clásicos).

Los autores desde su objetivo general, adoptaron y adaptaron, la taxonomía planteada por Barret (1968), a los siguientes niveles de comprensión lectora guiados hacia las matemáticas:

Comprensión literal. Donde el estudiante reconoce lo que el cuento le está explicando, teniendo en cuenta los siguientes aspectos: recuerda detalles de nombres de figuras geométricas, sucesos, tiempo, lugar y tipo de operaciones; ideas principales donde están explícitas las operaciones a realizar; secuencia de las mismas; causa y efecto de las operaciones matemáticas y rasgos de personajes, es decir, características explícitas de quién realiza la operación y con qué objetivo.

Comprensión inferencial. Se evidencia cuando el estudiante asocia los conceptos extraídos en el texto con los que ya posee; clasifica el tipo de operaciones que se presentan y cuáles puede emplear para llegar más fácilmente al resultado; esquematiza y planea una ruta de desarrollo de los problemas; resume el problema planteado en el cuento y sus posibles soluciones; detalla estrategias de solución a los problemas encontrados e identifica el tema matemático que está planteado en el cuento.
Los autores desde su objetivo general, adoptaron y adaptaron, la taxonomía planteada por Barret (1968), a los siguientes niveles de comprensión lectora guiados hacia las matemáticas. 
Para identificar el problema motivo de estudio, los investigadores emplearon los instrumentos: diario de campo de docentes y estudiantes, ya que es un registro narrativo que favorece la reflexión sobre la praxis
Lectura crítica o de juicio. El estudiante propone una solución sustentándola con el desarrollo de operaciones y argumentos válidos, para ello plantea hipótesis acerca de las posibles soluciones del problema matemático; determina si los problemas planteados en el texto, lo llevan a la adquisición real del concepto matemático y es capaz de juzgar, desde un punto de vista ético, la actitud y las acciones de los personajes.

Apreciación. Se está en este nivel cuando el estudiante analiza si lo que aprendió es útil o no para su vida académica y personal, se identifica con los personajes y las situaciones problemáticas que tuvo que resolver, entiende el concepto que el autor le está transmitiendo y es capaz de sustentar sus resultados de manera verbal y no verbal.

\section{Metodología}

\section{Participantes}

La unidad de análisis corresponde a 33 estudiantes de grado décimo A, de la Institución Educativa Magdalena, situada en la ciudad de Sogamoso (Boyacá), cuyas edades oscilan entre quince y dieciséis años: nueve de género femenino y veinticuatro masculino. El enfoque que se adoptó es el cualitativo, de corte crítico social, ya que, uno de los aspectos más importantes de este, es el hecho práctico, que el investigador se convierte en el instrumento principal de recolección y análisis de datos. Esto lleva a ser muy riguroso con la búsqueda de la teoría que permite alcanzar el objetivo planteado.

\section{Diseño y procedimiento}

Para identificar el problema motivo de estudio, los investigadores emplearon los instrumentos: diario de campo de docentes y estudiantes, ya que es un registro narrativo que favorece la reflexión sobre la praxis, aporta información relevante para la investigación, permitiendo así evaluar el proceso que se estaba llevando a cabo, comparando los resultados obtenidos con referentes teóricos (Vásquez, 2002). Los elementos que constituyeron el diario de campo, fueron: descripción de la actividad, análisis y acciones interventoras. Pruebas documentales que brindaron la posibilidad de abordar desde una perspectiva integral y participativa problemáticas sociales, en este caso, educativas, que requerían algún cambio o desarrollo. Aquí se partió de un diagnóstico de una situación observada, se identificó y valoró una alternativa de acción, para finalmente, elaborar un plan de cambio. Este instrumento requirió de un alto compromiso de los investigadores, ya que era necesario estar en constante análisis y evaluación. Este tipo de prueba comprendió las tres etapas: Encuadre, donde se identificó a los participantes, se planteó el objetivo y una agenda de trabajo; Diagnóstico, se elaboraron las preguntas del taller y se examinó la viabilidad del taller propuesto; y, por último, se estructuró y concertó el Plan de trabajo (aplicación de la prueba) (Taylor \& Bogdan, 2000).

Las pruebas se realizaron en tres momentos: Prueba diagnóstica, en donde se diseñó un cuestionario que 
planteó un problema matemático de aplicación del teorema de Pitágoras, con diez preguntas, que buscaban identificar en qué nivel de comprensión lectora se encontraban los estudiantes.

Prueba intermedia, allí se explicaron los niveles y cómo estaba conformado cada uno, permitiendo al estudiante analizar su propio proceso lector, así como también la definición del cuento como recurso didáctico en el desarrollo de sus habilidades lectoras. Para esta prueba, se seleccionó el cuento "Dido y la piel de buey", el cual es adaptación de la leyenda presente en la Eneida de Virgilio. Esta prueba dirigida permitió que el estudiante hiciera preguntas acerca del tema, siendo estas resueltas por los docentes.

Prueba final, que permitió evaluar el nivel de comprensión en el que se encontraban los estudiantes, después de haber empleado el cuento como eje motivador, mediante la adaptación del cuento "Viernes Cultural: Arquímedes y las Comunicaciones" (Perich, 2008), en donde el estudiante debía ser capaz de resolver la problemática por él mismo.

Estas últimas dos pruebas contenían cuentos que giraban en torno a una idea principal, de una situación cotidiana; en ellos se evaluaron los cuatro niveles de comprensión lectora a través de diferentes tipos de preguntas. En esta dirección, se presentan los resultados de la prueba final, con su respectivo análisis.

\section{Resultados}

Los resultados presentados hacen parte del desempeño alcanzado por los estudiantes de grado décimo A, en cada uno de los niveles de comprensión lectora en la prueba final, después de haber empleado el cuento como recurso didáctico. Para la selección de las preguntas que conformaron la prueba final, se tuvo en cuenta los niveles propuestos por los investigadores, con el fin de brindar al docente de matemáticas una herramienta lúdica, en donde no solo evaluará la resolución de un problema matemático, sino también la capacidad del estudiante para llegar a ese resultado y la trascendencia y uso que este le da en su vida diaria. El anterior análisis mostró que la gran mayoría de los estudiantes alcanzaron un nivel de comprensión positivo, lo que indica que el uso del cuento motiva, incentiva y desarrolla en ellos la capacidad lectora, haciendo que respondan correctamente, comprendan el problema que se les formula y busquen la mejor forma de solucionarlo.

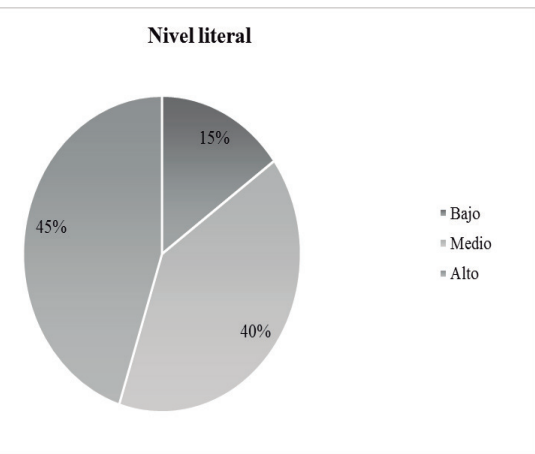

Figura 1. Desempeño nivel literal prueba final.

Fuente: Elaboración propia. 
En la figura 1, se presenta el porcentaje de estudiantes en cada uno de los desempeños bajo, medio y alto, correspondientes al nivel literal. Los estudiantes por medio de textos literarios como el cuento, pueden extraer más fácilmente la información contenida. Manifiestan que recuerdan detalles, extraen la idea principal, siguen una secuencia y reconocen la causa y efecto de las operaciones matemáticas. Durante el análisis de los resultados del nivel literal de acuerdo con los criterios evaluativos propuestos por los investigadores, se encontró progreso en el nivel comprensivo de los estudiantes. Solo la sexta parte de los alumnos obtuvo desempeño bajo, y los demás se ubicaron en medio y alto. Los investigadores reconocieron que los estudiantes se motivaron más y desarrollaron la prueba activamente y con gusto: se evidenció que el uso del cuento motiva al estudiante a la vez que lo sumerge en un mundo imaginativo, donde se enfrenta a diferentes aventuras, va adquiriendo y solucionando problemas matemáticos, además de resolver la situación inicial de la apatía hacia las matemáticas.

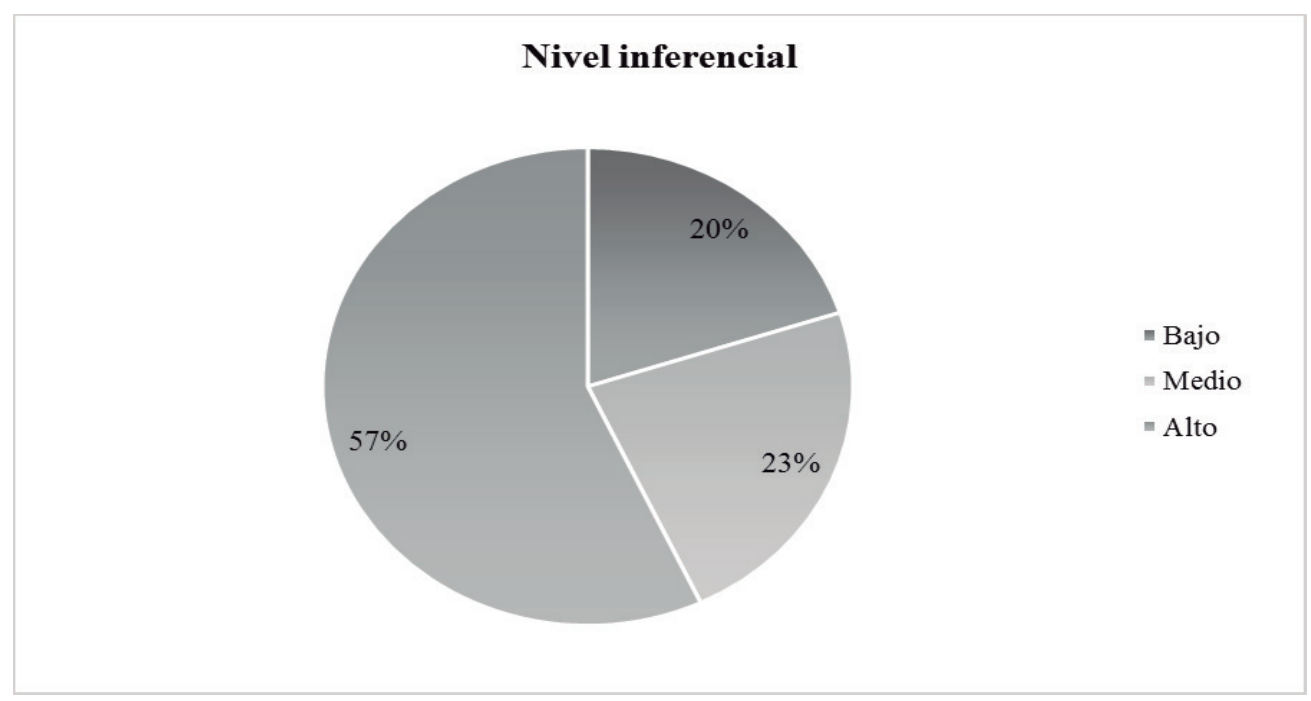

Figura 2. Desempeño nivel inferencial prueba final.

Fuente: Elaboración propia.

En relación con el nivel inferencial, en la figura 2 se observa que la proporción de estudiantes que desarrollaron el nivel alto es mayor. Además, se evidenció que el estudiante a través del cuento desarrolla su nivel inferencial, siendo capaz de activar sus conocimientos previos, de definir con sus propias palabras conceptos matemáticos, y formular estrategias de solución a los problemas planteados. La actividad los motivó a aprender e investigar más sobre temas que ya habían visto. 


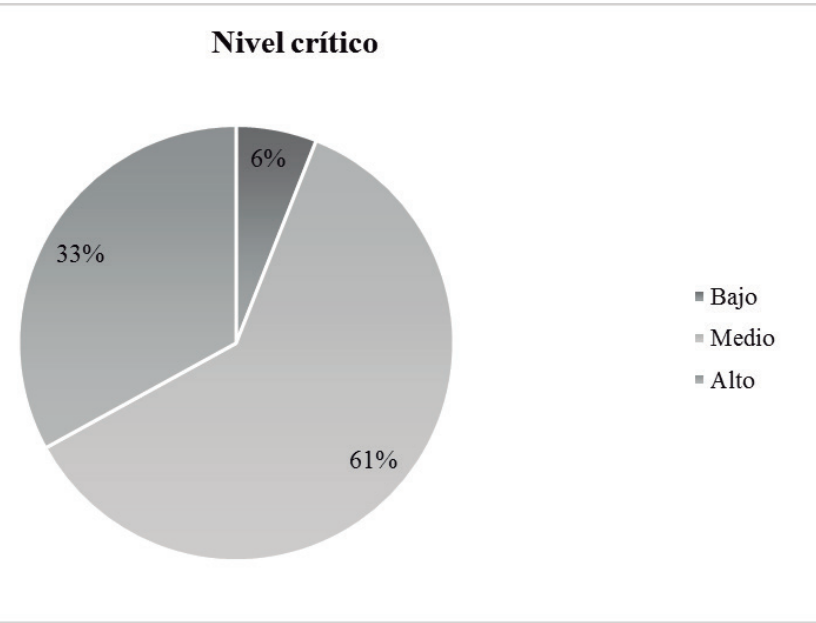

Figura 3. Desempeño nivel crítico prueba final.

Fuente: Elaboración propia.

En la figura 3, se evidencia que una mínima parte de la unidad de análisis obtuvo un desempeño bajo: la mayoría un desempeño básico, y el restante un desempeño alto. Durante la aplicación de la prueba final, se observó que los estudiantes llegaron por su cuenta a la solución del problema planteado, demostraron seguridad, agrado y responsabilidad ante el trabajo asignado, pues desarrollaron la capacidad de comprender y entender el problema planteado en el cuento; este les permitió tener mayor habilidad para la interpretación del texto escrito. Los estudiantes comentaron los sucesos del cuento y dieron sus puntos de vista de manera coherente y los expusieron en sus diarios de campo.

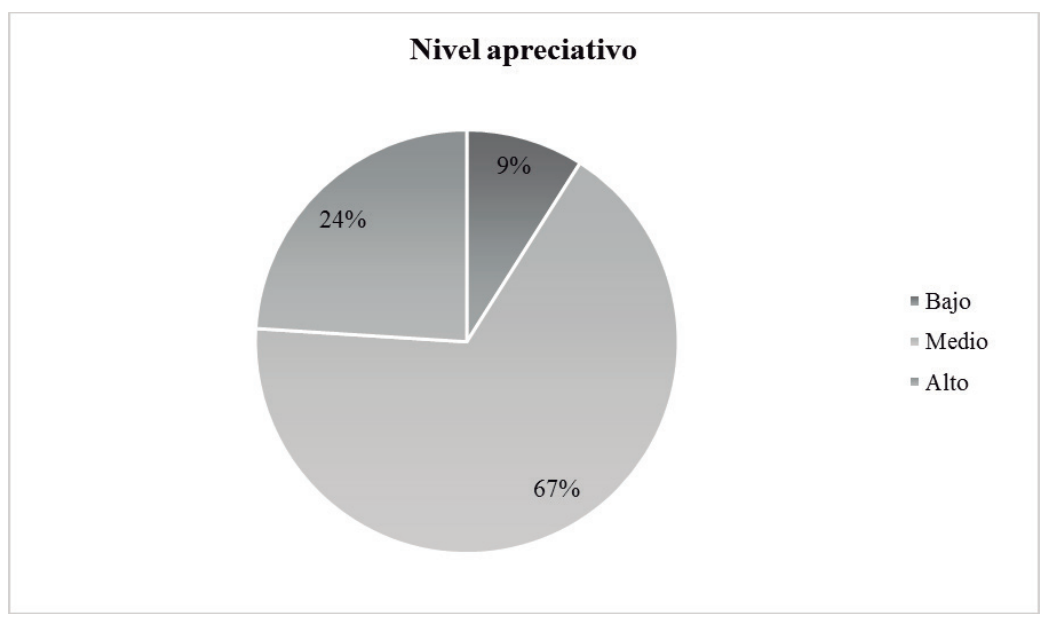

Figura 4. Desempeño nivel apreciativo prueba final.

Fuente: Elaboración propia. 
En la figura 4, se evidencia que los estudiantes potenciaron notablemente su comprensión en los cuatro niveles propuestos por los investigadores. El avance que presentaron los estudiantes en el transcurso de esta prueba fue evidente, fueron capaces de determinar si los conceptos adquiridos a través del cuento les eran útiles en su vida personal y académica. En su mayoría, entendieron el tema que se les estaba enseñando y se identificaron con los personajes. El cuento matemático les permitió resolver problemas presentes en diferentes contextos y campos de su vida, a la vez que admitió un cambio de actitud positivo hacia la clase de matemáticas.

A nivel general, se puede decir que el desempeño de los estudiantes frente a la prueba de comprensión lectora final, tendió a presentarse en diferentes proporciones, siendo mayor el desempeño en los niveles literal e inferencial, como se muestra en la figura 5.

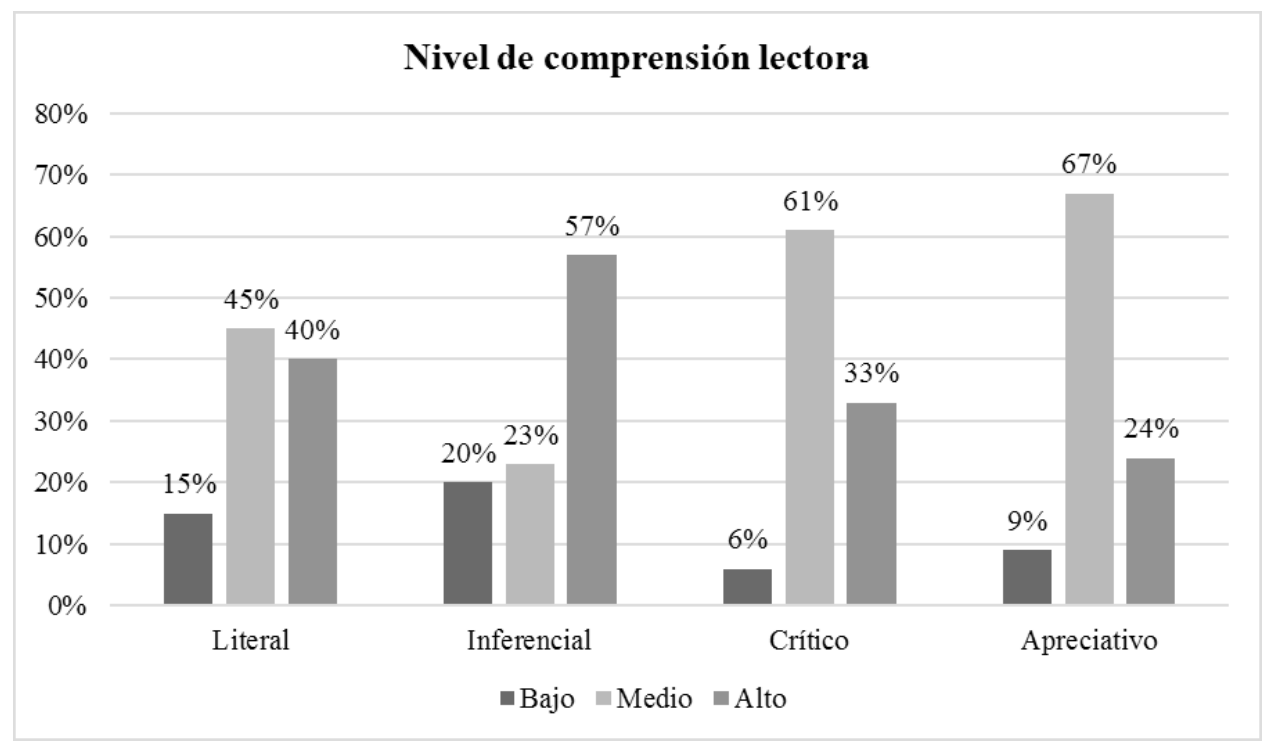

Figura 5. Contraste de desempeño prueba final.

Fuente: Elaboración propia.

\section{Discusión}

El objetivo central del estudio fue desarrollar los niveles de comprensión lectora en matemáticas de los estudiantes de grado décimo A. Se empleó el cuento como eje motivador para permitir que el estudiante abordara las temáticas con mayor disposición, y por tanto, con mayor probabilidad de alcanzar altos desempeños académicos.

Detrás del objetivo educativo, se buscaba que la estrategia empleada fuera motivante y que generará mayor aceptación y disposición de los 
estudiantes hacia la lectura, llevándolos a aplicar los conocimientos adquiridos en su cotidianidad, "Para tener éxito en la vida, los estudiantes deben aplicar estrategias de resolución de problemas más allá de las materias en que fueron aprendidas" (Martins, 2014). A la hora de resolver la prueba, se observó en los estudiantes reacciones de interés y aceptación frente al contenido textual y visual del cuento. Frente a los tipos de pregunta utilizados, se pudo concluir que la mezcla de imágenes, historias, problemas matemáticos y transversalidad de asignaturas, permitió que la prueba fuese abordada con mayor facilidad y agrado.

A través de la triangulación de la información recolectada con los instrumentos, se encontró que, en el nivel literal, el éxito de la solución de problemas matemáticos consiste en la capacidad que tiene el estudiante de identificar los datos más importantes. El cuento permitió que el estudiante se concentrara en estos aspectos y que lograra, de manera más fácil, identificar el problema planteado. Según el estudiante N5, "Se me hizo más fácil responder preguntas sobre qué tipo de operación desarrolló y cuál es el personaje, etc. a través de una historia, ya que me parece más agradable a la vez que aprendo". Partiendo de esto, Blanco y Blanco (2009) afirman que el cuento constituye un elemento motivador en el aula, generando una actitud más favorable en los alumnos de cara a las matemáticas y facilitando la comprensión de conceptos contenidos en el mismo.
En el nivel inferencial, se encontró que la actividad los motivó a aprender e investigar más sobre temas que ya han visto. Blanco y Blanco (2009) señalan la eficacia de los cuentos matemáticos, argumentando que permiten relacionar contenidos matemáticos con los conocimientos previos de los alumnos y evaluar las nociones adquiridas. Es decir, el estudiante se sumerge en un sinnúmero de situaciones que le permiten imaginar la historia que se le presenta, a la vez que va resolviendo problemas matemáticos, se enfrenta a reflexiones sobre temas y conceptos que ya posee, para plantearse posibles soluciones y llegar al final. Para complementar, el estudiante N13 argumentó: "Es más fácil recordar temas que ya poseo a través de un cuento porque si me preguntas qué es esto o lo otro, se me dificulta más responder".

Se manifiesta que, a los estudiantes les gusta los cuentos matemáticos, así la clase no es tan monótona y tradicional. El estudiante N15 manifestó: "me concentro tanto en la lectura que empiezo a pensar en cuál sería la mejor manera para que mi personaje llegue rápido a la solución del problema”. Con base en esto, se puede concluir que las lecturas narrativas permiten que el estudiante desarrolle su nivel crítico, ya que hacen que formen parte de la historia, se interrogue sobre qué debe hacer, cuál es el mejor camino para encontrar el final, en conclusión, comprenda lo que lee. Estudiante N30: "me gusta más desarrollar problemas matemáticos a través de cuentos, yo mismo busco la solución del problema
A través de la triangulación de la información recolectada con los instrumentos, se encontró que, en el nivel literal, el éxito de la solución de problemas matemáticos consiste en la capacidad que tiene el estudiante de identificar los datos más importantes. 
sin estar esperando a que el profe me diga qué hacer [...] puedo ponerme en la posición del personaje y juzgar si lo que hizo está bien o mal”.

En el último nivel, se concluye que el cuento matemático les permite resolver problemas presentes en diferentes contextos y campos de su vida. Los cuentos son una herramienta útil en las clases de matemáticas, dado que permiten que los alumnos comprendan los contenidos trabajados, los utilicen de manera eficiente y los empleen en otros contextos de aprendizaje. "No sabía que algunas aplicaciones de los inventos de Arquímedes podía encontrarlos en mi casa..." estudiante N26. Se debe llevar al estudiante a que aplique lo aprendido en el aula en todas las áreas de su vida.

Este estudio evidencia que los estudiantes de grado décimo A, lograron desarrollar los niveles de comprensión lectora, mostrando que es posible utilizar el cuento como recurso, así como lo afirma Marín (1999): se puede considerar el cuento como una herramienta para disuadir a los alumnos de la idea de las matemáticas como una materia aburrida, aumentando la motivación de estos hacia esta disciplina. Es el reto de los docentes para la utilización de recursos que les permitan ejercitar las habilidades que poseen los estudiantes, y que potencialicen los procesos de enseñanza-aprendizaje en las diferentes áreas.

\section{Conclusiones}

El estudio, titulado El cuento de las matemáticas, fortaleció la comprensión lectora de los estudiantes de grado décimo A, de la Institución Educativa Magdalena. Los investigadores demostraron que, a través del recurso el cuento, se pueden desarrollar los niveles de comprensión lectora en matemáticas.

La inserción de un recurso narrativo, en este caso el cuento, puede llevar a fortalecer la comprensión lectora en matemáticas a través de los cuatro niveles (literal, inferencial, crítico y apreciativo). Esta interrelación entre el cuento matemático y los niveles, evidencia que el uso de un recurso que despierte la imaginación, la interacción del estudiante con los personajes y los problemas asociados con su vida, desarrolla de manera coherente, organizada y progresiva conocimientos o saberes matemáticos; la forma de prueba documental permite al docente crear, recrear e innovar su práctica pedagógica constantemente. Se ejemplifica esta innovación didáctica con la implementación del género literario el cuento, se fortalece la comprensión lectora en matemáticas en la mayoría de los estudiantes de grado décimo A de la Institución Educativa Magdalena de Sogamoso.

Los investigadores recurrieron a la estrategia didáctica del cuento a través de pruebas documentales (diagnóstica, intermedia y final); una, dirigida; las otras dos, individuales. Los estudiantes se convirtieron en protagonistas del relato presentado cuando lograron comprender lo que se les estaba preguntando (literal), relacionaron los temas que ya sabían con los nuevos 
que el texto les presentaba (inferencial), realizaron razonamientos (crítico), $\mathrm{y}$, por último, cuando empezaron a usar la temática aprendida en diferentes contextos (apreciativo). Los alumnos opinaron, presentaron posibles soluciones a diferentes problemas matemáticos, llegando así a resolver el conflicto del cuento. El aula de matemáticas se convirtió en un escenario dinámico, entretenido y enriquecedor de enseñanza de saberes significativos, en donde, el estudiante fue el actor principal.

La comprensión lectora se desarrolló gracias a la aplicación de la estrategia que contenía los cuatro niveles dentro de un texto literario. Se puede concluir que, enseñar a leer comprensivamente puede realizarse de manera transversal, en este caso, no solo ayudó a comprender temáticas matemáticas, sino también aportó al desarrollo de la lengua castellana. De esta manera, se puede decir que el estudiante de grado décimo $A$ se desempeñará mejor en cada una de estas asignaturas, porque la transformación vista en la clase de su docente le ayudó a cambiar el concepto que tenía sobre su asignatura. La comprensión lectora, sin duda alguna, ayuda a contribuir en el mejoramiento de los resultados obtenidos en pruebas SABER y PISA, aún más si el docente se atreve a proponer nuevas estrategias que motiven al alumno a analizar y comprender lo que lee y para qué le sirve lo que aprende en el colegio; esto se logró en buena medida en la Institución Educativa Magdalena, dado que se decidió innovar y presentar las experiencias de aula de una manera más lúdica y propositiva, evidenciando el cambio de actitud de los estudiantes.

En el nivel literal, se encontró que, al principio, 17 de los 33 estudiantes tenían una valoración baja; y 4, una valoración alta. Pero, después de la aplicación del recurso, se evidenció que 5 de los estudiantes obtuvieron una valoración baja; y 15, valoración alta; lo cual demuestra que este nivel se desarrolló de manera positiva.

En cuanto al nivel inferencial, en la prueba diagnóstica se identificó que 3 alumnos se encontraban en alto; pero, en la prueba final, 19 estudiantes alcanzaron el nivel alto, lo que demostró una mejora considerable. Los estudiantes en el nivel crítico también mostraron un avance ya que en la prueba diagnóstica solo 4 alcanzaron un nivel alto en comparación con la prueba final, en donde 11 de ellos fueron capaces de proponer una solución al problema presentando argumentos válidos. Finalmente, en el nivel apreciativo se reconoce un incremento en la prueba final frente a la diagnóstica, ya que 27 estudiantes ahora determinaron si lo que aprendieron era útil o no para su vida diaria en contraste con 3 que lo hacían antes. Estos resultados afirman que, mediante la aplicación del recurso el cuento, se obtienen resultados favorables en cuanto al fortalecimiento de la comprensión lectora y el efecto positivo del desarrollo de sus niveles (literal, inferencial, crítico y apreciativo).
En el nivel literal, se encontró que, al principio, 17 de los 33 estudiantes tenían una valoración baja; y 4, una valoración alta. 


\section{Referencias}

Alonso, L. A., Parada, R. A., \& Chaparro, A. Z. (2016). El razonamiento como eje transversal en la construcción del pensamiento lógico. Praxis \& Saber, 7(14), 219-143. https://doi.org/10.19053/22160159.5224

Barrett, T. C. (1968). Taxonomy of Cognitive and Affective Dimensions of Reading Comprehension. En Theodore Clymer, "What is Reading?": Some Current Concepts, En Helen M. Robinson (Ed.) Innovations and change in Reading Instruction, Sixty-seventh Yearbook, National Society for the Study of Education, Part II. Chicago, University of Chicago Press.

Marín, R. M. (1999). El valor del cuento en la construcción de conceptos matemáticos. Didáctica de las matemáticas, volumen, (39), 27-38.

Martins, A. (2014, abril 01). Los estudiantes de América Latina "no resuelven problemas de la vida real”. BBC Mundo. Recuperado de http://www.bbc. com/mundo/noticias/2014/04/140401_pisa_problemas_vida_am\ (2008). Las Aventuras Matemáticas de Daniel. Chile: Editorial Impacto.

Pinzás, J. (1995). Leer pensando. Lima: Asociación de Investigación Aplicada y Extensión Pedagógica Sofía Pinzas.

Porlán, R. (1993). Constructivismo y Escuela. Hacia un modelo de enseñanza-aprendizaje basado en la investigación. Sevilla, España: Díada Editorial S.L.

Salas, P. (2012). El desarrollo de la comprensión lectora en los estudiantes del tercer semestre del nivel medio superior de la Universidad Autónoma de Nuevo León (tesis de maestría). Universidad Autónoma de Nuevo León, San Nicolás de los Garza, México.

Taylor, S., \& Bogdan, R. (2000). Introducción a los métodos cualitativos de investigación. Barcelona, España: Paidós ibérica S.A.

Vásquez, F. (2002). El diario de campo una herramienta para investigar en preescolar y primaria. Serie formación de maestros Bogotá DC, Proyecto de restructuración de escuelas normales, 111. 